\title{
Effects of Total and Partial Sleep Deprivation on Reflection Impulsivity and Risk-Taking in Deliberative Decision-Making
}

This article was published in the following Dove Press journal: Nature and Science of Sleep

\author{
Federico Salfi (D) \\ Marco Lauriola ${ }^{2}$ \\ Daniela Tempesta ${ }^{1}{ }^{1}$ \\ Pierpaolo Calanna (1D ${ }^{3}$ \\ Valentina Socci' \\ Luigi De Gennaro (D) ${ }^{4}$ \\ Michele Ferrara (D) \\ 'Department of Biotechnological and \\ Applied Clinical Sciences, University of \\ L'Aquila, L'Aquila, Italy; ${ }^{2}$ Department of \\ Social and Developmental Psychology, \\ Sapienza University of Rome, Rome, Italy; \\ ${ }^{3}$ Department of Dynamic and Clinical \\ Psychology, Sapienza University of Rome, \\ Rome, Italy; ${ }^{4}$ Department of Psychology, \\ Sapienza University of Rome, Rome, Italy
}

Correspondence: Michele Ferrara Department of Biotechnological and Applied Clinical Sciences, University of L’Aquila, Via Vetoio (Coppito 2), Coppito $67100, A Q$, Italy

Email michele.ferrara@univaq.it
Study Objectives: To evaluate the effects of total and partial sleep deprivation on reflection impulsivity and risk-taking in tasks requiring deliberative decision-making processes.

Participants and Methods: Seventy-four healthy young adults were selected to participate in two independent experiments, each consisting of a crossover design. In Experiment 1, 32 participants were tested after one night of regular sleep (RS), and after one night of total sleep deprivation (TSD). In Experiment 2, 42 participants were tested following five nights of RS and after five nights of partial sleep deprivation (PSD), implying five hours of sleep per night. In both the experiments, two deliberative decision-making tasks were administered, involving different decision-making constructs. The Mosaic Task (MT) assessed reflection impulsivity, the tendency to gather information before making a decision. The Columbia Card Task cold version (CCTc) evaluated risk-taking propensity in a dynamic environment.

Results: Unlike TSD, PSD led to an increment of reflection impulsivity and risk-taking. Nevertheless, analyses taking into account the individuals' baseline (RS) performance showed consistent results between the two experimental sleep manipulations. Participants who gathered more information to make decisions in the MT when well-rested, then relied on less evidence under sleep loss, and more cautious participants in the CCTc tended to make riskier decisions.

Conclusion: Results pointed to differential consequences of sleep deprivation depending on the habitual way to respond during decision-making involving deliberative reasoning processes. Results were interpreted according to a putative interaction between sleep loss effect and individual difference factors.

Keywords: sleep deprivation, sleep restriction, reflection impulsivity, risk-taking, deliberative decision-making, individual differences

\section{Summary}

We investigated for the first time the effect of both total and partial sleep deprivation on tasks involving specifically the deliberative component of decision-making. Our results show that the effects of prolonged sleep restriction are more detrimental than those of one night of total sleep deprivation. Furthermore, our findings point to substantial inter-individual differences in the consequences of sleep loss. An inadequate amount of sleep differentially impacted participants' performance depending on their habitual decision behavior when well-rested. Under the effects of sleep loss, people habitually more reflective and cautious become more impulsive and 
prone to risk-taking during decision-making based on deliberative reasoning. The current results highlight the importance of considering individual differences in sleep deprivation protocols.

\section{Introduction}

A large percentage of people in most industrialized nations regularly sleep inadequately, outlining a real sleep loss epidemic. ${ }^{1}$ The link between sleep deficiency and health problems is widely documented. ${ }^{2}$ Moreover, sleep loss compromises basic cognitive functions, such as psychomotor vigilance, ${ }^{3}$ attention, and working memory. ${ }^{4}$ Negative consequences of an inadequate amount of sleep also involve higher-order cognitive processes such as decision-making. Behavioral studies showed that sleep deprivation is linked to impairment in the quality of decision-making across multiple domains, reducing the ability to make good judgments. ${ }^{5}$ An increased propensity to take risks following sleep deprivation has been shown in decision-making under uncertainty. ${ }^{6,7}$ Furthermore, sleep loss affects economic preferences $^{8}$ and has an effect on impulsive decisionmaking. ${ }^{9,10}$ Two competing systems were proposed to underlie the decision-making processes. ${ }^{11}$ One system accounts for "hot" decision-making, involving "gut feelings" and emotions. ${ }^{12}$ The other system, phylogenetically younger, is based in deliberative thought processes, relying on analytic evaluations of risks/benefits. This process can be referred to as "cold" decision-making. ${ }^{13,14}$

The decision-making tasks commonly employed in the sleep literature hinge upon both hot and cold processes, hindering the identification of specific components of decision-making compromised by an inadequate amount of sleep. ${ }^{15}$ The issue is evident in the research involving the Iowa Gambling Task ${ }^{16}$ (IGT), which has been widely used to assess risky choice behavior under sleep deprivation. ${ }^{10}$ This task makes it difficult to discern if sleep loss affects only one's risk attitude or subjective probability formation. ${ }^{17}$ The same reasoning applies to the studies evaluating the consequences of sleep deprivation on social-economic tasks (eg, Ultimatum Game ${ }^{18,19}$ ). Although the affective component seems to be predominant in these tasks, the decisions also involve deliberative processing. ${ }^{20,21}$

The issue of task impurity recommends caution in the interpretation of the available results, ${ }^{22}$ preventing to draw unambiguous conclusions about the relationship between sleep loss and decisional processes. ${ }^{15}$ Given that real-world decision-making is based on complex interactions between hot and cold processes, ${ }^{23}$ it is essential to examine them in a condition of sleep loss, separately. Addressing different aspects of cognitive decision-making is needed because sleep deprivation does not uniformly affect the multiple components underlying cognition involved in decisional processes. $^{24,25}$

In the current study, we used two tasks that predominantly involve cold processes, but assessing different decision-making dimensions: the Mosaic Task (MT) and the Columbia Card Task cold version (CCTc). The MT is equivalent to the Beads Task, presenting differences only in the graphical interface (for details see "Participants and Methods"). The Beads Task is a validated probabilistic reasoning task, ${ }^{26}$ which has been almost exclusively used in clinical research to evaluate a specific subtype of "cold" decisional impulsivity termed reflection impulsivity. ${ }^{27-30}$ This task was selected to assess the consequences of sleep loss on the tendency to gather increasing evidence to achieve a decision in a forced dichotomous choice, considering that sleep deprivation is capable of reducing the weight placed on new pieces of information in a decision task similarly based on Bayesian updating. ${ }^{17}$ The CCTc is a risk-taking task developed to assess predominantly deliberative decision-making processes ${ }^{31}$ mostly requiring a rule-based elaboration that involves working memory and mental calculation. ${ }^{32}$ In the view of the well-known increment of risk propensity following sleep deprivation, ${ }^{33,34}$ the choice of this task was aimed at evaluating the impact of an inadequate amount of sleep on decision-making strategies that do not engage affective components. ${ }^{35}$

Most studies in sleep literature on decision-making have used protocols involving total sleep deprivation (TSD), since it represents a more systematic approach to evaluate the consequences of sleep loss on cognitive functions, allowing to effectively control for potential confounding factors in a laboratory setting. ${ }^{33}$ On the other hand, prolonged partial sleep deprivation (PSD) certainly represents a more prevalent condition in industrialized societies (due to work requests, lifestyle or medical conditions), which exerts similar consequences on cognitive functioning as a period of TSD. ${ }^{36,37}$

Through two distinct experiments, this study aimed to evaluate the impact of both TSD and five consecutive nights of PSD (5 hours per night) on cold decisionmaking processes in healthy young adults. We hypothesized that sleep loss could reduce the amount of collected information before making decisions in MT, influencing the reasoning process based on the arrival of new 
information. Furthermore, we expected that both experimental sleep manipulations lead to an increased risk propensity in a purely deliberative risk task such as CCTc.

\section{Participants and Methods}

The study included two independent experiments, carried out at the Laboratory of Sleep Psychophysiology and Cognitive Neurosciences, Department of Biotechnological and Applied Clinical Sciences of the University of L'Aquila. Both the experiments have been approved by the Internal Review Board of the University of L'Aquila (Italy), carried out according to the principles established by the Declaration of Helsinki. Written informed consent was obtained from all participants before both the experiments.

\section{Experiment I: Total Sleep Deprivation Participants}

Thirty-two university students were prescreened to participate in the experiment (mean age $\pm \mathrm{SE}, 22.13 \pm 0.53 \mathrm{yr}$; 7 males). To check for the presence of sleep disorders, insomnia, and mood or anxiety disorders, each participant filled out the Pittsburgh Sleep Quality Index ${ }^{38,39}$ (PSQI), the Insomnia Severity Index ${ }^{40,41}$ (ISI), the Beck Depression Inventory ${ }^{42}$ (BDI-II) and the State-Trait Anxiety Inventory ${ }^{43}$ (STAI-T). Inclusion criteria were: a score $<6$ for the PSQI (group mean $\pm \mathrm{SE}, 3.03 \pm 0.26$ ), a score $<7$ for the ISI $(2.94 \pm 0.44)$, a score $<41$ for the STAI $(34.51 \pm 0.92)$ and a score $<14$ for the BDI $(5.30 \pm$ 0.64). All subjects had a habitual sleep duration of 7-8 hours per night, went to bed between 11:00 p.m. and midnight, and did not usually take naps during the day.

\section{Experimental Protocol}

A crossover design was used, involving two conditions, in a counterbalanced order, spaced one week apart (Figure 1).
One week before the day of the experiment, participants came to the laboratory to carry out a preliminary session, in which they received detailed instructions and familiarized themselves with the experimental tasks using a short demo of each task. Participants were recommended to maintain a regular sleep-wake cycle in the five days preceding both the conditions: we checked compliance using actigraphy, which confirmed the absence of significant differences in sleep amount and quality between the two conditions (see "Results" section).

In the regular sleep condition (RS), participants took part in the Testing phase (scheduled at 9:00 a.m.) after a regular sleep night spent at home, measured by actigraphic recording (see "Sleep Assessment").

In the total sleep deprivation condition (TSD), the subjects came to the laboratory at 9:00 p.m., where they spent the night awake. They were asked to spend the day preceding the deprivation night following their habits, avoiding to sleep during the day. This aspect was controlled through actigraphy.

During the deprivation night, light snacks were allowed, while activating beverages such as tea, coffee, and energy drinks, chocolate, alcohol, and medications inducing or contrasting sleepiness were banned. Regular smokers were required not to smoke more than one cigarette every hour. All participants were also imposed not to smoke or eat at least 30 minutes before the Testing phase. Additionally, lying down, sleeping, and performing intense physical activity was not allowed. Participants were permitted to briefly walk around, listen to music, read, watch the television, or use a computer. Overnight, two experimenters constantly monitored the research participants. Neon lamps illuminated the laboratory. Time information was available to research participants. The next morning, at 9:00 a.m., each subject participated in the Testing phase.

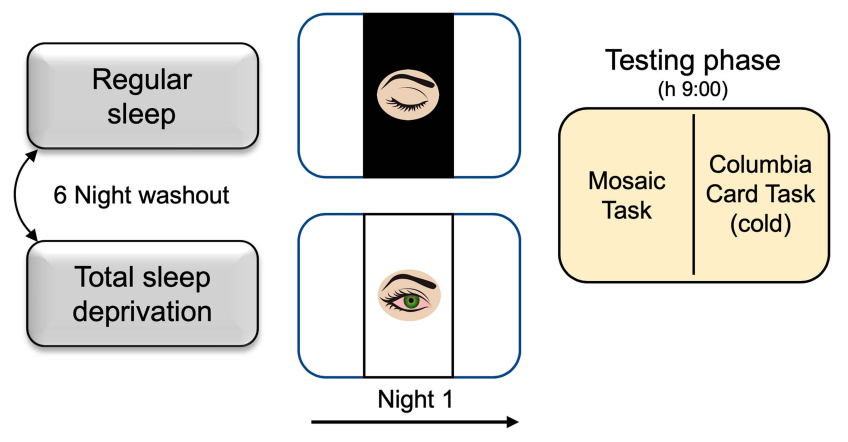

Figure I Experimental protocol of Experiment I.

Notes: Schematic representation of the experimental protocol of Experiment I that included regular sleep and total sleep deprivation conditions, presented in counterbalanced order and separated by one week. The Testing phase was scheduled the morning following the night of both conditions. 


\section{Sleep Assessment}

All participants wore an actigraph on the non-dominant wrist (AMI MicroMini Motionlogger; Ambulatory Monitoring, Ardsley, NY, USA) during the five days before the conditions (RS, TSD). Additionally, in the RS condition, the night preceding the Testing phase was controlled through actigraphy. Actigraphs were initialized by ACT Millennium software (Ambulatory Monitoring, Ardsley, NY, USA) in zero crossing mode to collect data in 1-min epochs. Collected data have been analyzed through Action W-2 software (Ambulatory Monitoring, Ardsley, NY, USA). Moreover, each morning all the participants completed a sleep diary to report their subjective sleep duration and sleep quality. Three variables, total sleep time (TST), sleep efficiency (EFF\%), and wake after sleep onset (WASO), were obtained by the actigraphic data. In the RS condition, the mean actigraphic variables $( \pm \mathrm{SE})$ calculated for the sleep night preceding the Testing phase were: TST $=443.79$ minutes $( \pm 7.73)$, $\mathrm{EFF} \%=94.58 \%( \pm 0.82)$, WASO $=19.03$ minutes $( \pm 3.60)$.

\section{Testing Phase}

At the beginning of the Testing phase, we assessed subjective sleepiness using the Karolinska Sleepiness Scale ${ }^{44}$ (KSS). Participants' subjective mood was measured using a computerized version of the Visual Analogue Scale ${ }^{45}$ (VAS): each participant rated his/her current mood along eight dimensions (happiness, sadness, tension, calmness, irritability, tiredness, energy and concentration). Accordingly, they pressed the left button of the mouse on a $200 \mathrm{~mm}$ long line, presented on the computer screen, between the extremes of "not at all" and "very much". We converted participant's ratings into values from 0 to 10 . We then calculated a Negative Mood Index (NMI), adding participant's scores on the items sad, tense, irritable, and happy and calm (reverse scored). A higher NMI reflected a more negative mood (range: 0-50). Finally, participants performed two decision-making tasks in a fixed order: the Mosaic Task (MT) and the Columbia Card Task, cold version (CCTc; details below). Since each task provides a final hypothetical cumulative earning, we informed participants that their score on each task would be subsequently used to rank their performance and published at the end of the entire data collection. Participants knew that if they scored in the top half of the ranking, this would be rewarded with two credits, while a score in the bottom half would be rewarded with one credit, only. This information aimed to motivate participants to do their best and maximize their performance. At the end of the study, all the participants were rewarded with the maximum coursecredits.

\section{Mosaic Task}

The Mosaic Task (MT) is inspired to the Beads Task, a probabilistic inference task used to evaluate reflection impulsivity, which is the tendency to collect and evaluate information before making a decision..$^{30,46-49}$ Participants must decide which jar a sequence of beads has been drawn from. ${ }^{50-52}$ Because the two jars typically used in a Beads Task were replaced by mosaics, and the beads by tiles, the task used in the current study was referred to as MT. Each mosaic had 100 tiles of two different colors in different proportions. In keeping with Garety et $\mathrm{al}^{50}{ }^{50}$ we set three difficulty levels defined by $30: 70,35: 65$, and 40:60 blue to red tiles ratio, respectively. Hereafter, we refer to these levels as easy, intermediate, and difficult, respectively.

The three levels were presented in a fixed order of increasing difficulty, and each one involved three repetitions of the task (trials). In each trial, the participant started with a fictitious budget of 400 points. After the subject pressed a button mixing the tiles, the two mosaics were hidden by a descending curtain. Then, the participant could draw some tiles. For each draw, 20 points were endowed from their budget, which was always shown in a panel next to the mosaics. The drawn tiles were randomly presented, according to the probabilities underlying the three difficulty levels. If the participant was accurate in deciding which of the two mosaics the tiles had been drawn from, the remaining budget was awarded to him/ her. In so doing, greater information seeking was penalized by more subtracted points. Accumulated points were also presented in a panel next to the mosaics during each trial. If the participant felt not ready to decide, he/she could go on drawing the next tile, and so forth, up to a maximum of twenty tiles. The participant's goal was to end up with the highest gain across all trials. The number of tiles drawn before deciding (Draw to Decide, DTD) was the main dependent variable recorded in each trial.

\section{Columbia Card Task, Cold Version}

The Columbia Card Task ${ }^{31}$ is a computerized card game used for assessing behavioral risk-taking tendencies in a dynamic environment. There are two different versions of the task, known as "hot" and "cold". In this study, we used the cold version (CCTc), which was devised to assess 
deliberative decision processes, hinging upon working memory and mental calculations. The peculiarity of the cold version is that no gain-loss feedback is given at the end of each game round (trial), and the final balance is revealed only at the end of the entire game. We administered a shortened version consisting of 27 trials instead of the 54 trials used in previous research. ${ }^{31}$ In each trial, the participants were presented with 32 face-down cards, arrayed in four rows of 8 cards each, and the participant selected how many cards he/she wanted to turn over by clicking on a number between 0 and 32 at the top of the screen, considering that each turned card corresponded to a precise gain amount in each trial. Every trial started with a score of 0 points. Participants were informed that they could lose a variable number of points if they encountered a losing card within the number of cards they had decided to turn over. The aim was to earn as many points as possible, and in each trial, the participants were informed (at the top of the screen) about: the gain amount per turned card $(10,20$, or 30 points), the number of loss cards $(1,2$, or 3 ), and the penalty if a loss card is encountered (250, 500 , or 750 points). Indeed, the 27 trials represent a full factorial combination of the 3 levels of the 3 factors mentioned above. The variation of these factors is a crucial feature of the CCTc, allowing to evaluate how available information can influence risky choices. The presentation order of the game rounds was randomized for each participant.

In the original $\mathrm{CCT}$, the losing cards were always the last in the trial, since it is improbable that research participants will become aware of this feature. ${ }^{31}$ However, considering that we planned to administer the task twice, the cards in each trial were shuffled, and the loss card could be encountered at any stage during the task. Previous research has shown that risk-taking tendencies can be reliably assessed using the unrigged version of the task. $^{53,54}$ Because turning over more cards conferred greater potential reward, but also higher risk, the CCTc main index of risk-taking was the number of turned cards in each trial (Turned Cards, TC), with higher scores indicating greater risk-taking.

\section{Data Analysis}

The effects of total sleep deprivation on perceived sleepiness (KSS) and negative mood index (NMI) scores have been assessed using paired-sample $t$-tests, comparing RS and TSD conditions. Likewise, paired-sample $t$-tests were used to compare the two conditions on the mean of sleep diary
(S-TST, S-WASO) and actigraphic variables (TST, EFF\%, WASO) recorded during the five nights preceding each condition. A p-level of $<0.05$ was considered significant.

To test hypotheses on the association of one night of sleep deprivation with decision-making in the Mosaic Task, DTD was analyzed at the trial level without aggregation. This approach allowed us to take the full response patterns into account, without averaging over individual trial-by-trial choices. DTD variable was tagged for each participant, to coding the experimental condition (RS, TSD) and the difficulty level of the task (easy, medium, difficult). Likewise, the CCTc scores were also analyzed at the trial level without aggregation to test hypotheses on the association of one night of sleep deprivation with risktaking. Trial-by-trial TC scores were tagged to coding the following variables for each participant: experimental condition (RS, TSD), gain amount (10, 20, 30 points), number of loss cards $(1,2,3)$, and loss amount $(250,500$, 750 points).

The analyses of MT and CCTc scores were performed using the lme4 R package, ${ }^{55,56}$ which provides functions for fitting and analyzing mixed models. Models were fitted using REML, and p-values were derived using the Satterthwaite approximation. ${ }^{57}$ For the MT, the model included the following predictors: experimental condition (RS, TSD), difficulty level (easy, medium, difficult), and the interactions among these factors. For the CCTc, the model included the main effects of experimental conditions (RS, TSD), gain amount (10, 20, 30 points), number of loss cards $(1,2,3)$, loss amount $(250,500,750$ points $)$ and the interactions among these factors (omitting interactions among the following CCTc factors: gain amount, number of loss cards and loss amount). All the analyses taking into account "gender" as a between-subject factor did not yield any significant main effect or interaction. Therefore, this factor was collapsed and is not reported in the results. Of note, mixed-model analyses included a random intercept per participant to account for the repeated-measures nature of the data and the hypothesized variability among participant responses. Importantly, although the main results were obtained from the two models described above, to clarify and isolate the effects observed in the omnibus models, we ran specific follow-up models reported in the "Results" section (omitting the description of main effects and interactions already commented for the main models). For significant effects, Bonferroni post hoc tests were carried out, and the level of significance was always set at $\mathrm{p}<0.05$. 


\section{Experiment 2: Partial Sleep Deprivation} Participants

Forty-five subjects were selected from a university student population to participate in the experiment. Three subjects were discarded due to technical failures. Consequently, the final sample comprised forty-two participants (mean age \pm SE, $24.22 \pm 0.62 \mathrm{yr} ; 21$ males). Each participant filled out the same screening questionnaires as in Experiment 1, with the same inclusion criteria (PSQI: group mean \pm SE, $3.62 \pm$ 0.22 ; ISI: $3.22 \pm 0.35$; STAI-T: $33.40 \pm 0.99$; BDI: $5.78 \pm$ 0.67). All participants declared to have a habitual sleep duration of $7-8 \mathrm{~h}$ per night, went to bed between midnight and 1:00 a.m., and did not have daytime nap habits.

\section{Experimental Protocol}

As in Experiment 1, all the subjects participated in two conditions, held during two consecutive weeks in a counterbalanced order (see Figure 2).

Similar to Experiment 1, a preliminary session was scheduled the week before the start of the experiment, in which participants received instructions on the two experimental tasks and participated in two short demo versions of them, to ensure a full understanding of the tasks the testing day.

In the regular sleep condition (RS), participants were allowed to sleep for five consecutive nights (from Sunday to Thursday) according to their sleep habits. In the other week, a maximum of 5 hours of sleep per night was imposed, for five consecutive nights (from Sunday to Thursday) [partial sleep deprivation condition (PSD)]. In the PSD condition, participants were instructed to go to bed approximately at 2:00 a.m. and to wake up at 7:00 a.m. Two washout nights were scheduled between the two experimental conditions. The Testing phase was carried out on the Friday morning of both weeks (about 9:00 a.m.). Participants were recommended not to smoke or eat at least 30 minutes before each Testing phase, and they were requested not to increase their habitual intake of activating beverages (tea, coffee, energy drinks), alcohol, medications, and cigarette consumption throughout the experimental protocol.

In both the conditions, participants had to warn the experimenter, through a text message, of the moment they went to bed, turned off the lights to sleep, woke up, and got out of bed. Constant telephone monitoring was carried out through calls and text messages to control the reliability of this information. During the night, the participants were recommended to keep their telephone near the bed, with the ringtone activated, so that the experimenters could wake them up at the scheduled time if they had not received the "good morning" text message. Daytime napping was not allowed through the entire experimental protocol. This aspect was controlled by an expert, who visually checked all the actigraphic recordings (see "Sleep Assessment" paragraph), also verifying the reliability of falling asleep and awakening times declared by the participants through sleep diaries and text messages.

\section{Sleep Assessment}

To obtain an objective assessment of sleep and to control participants' compliance with the experimental protocol, all participants wore a Geneactiv accelerometer (ActivInsights Ltd., Kimbolton, UK). The accelerometer was worn on the non-dominant wrist from the Sunday morning of the first condition, and for the entire duration of both the experimental conditions. The Geneactiv accelerometer is a reliable tool for evaluating sleep in adults. ${ }^{58}$ Devices were initialized by Geneactiv PC software (version 3.2, ActivIn-sights Ltd., Kimbolton, UK) with measurement frequency set to $50 \mathrm{~Hz}$. Geneactiv data were uploaded to the computer using the same software.

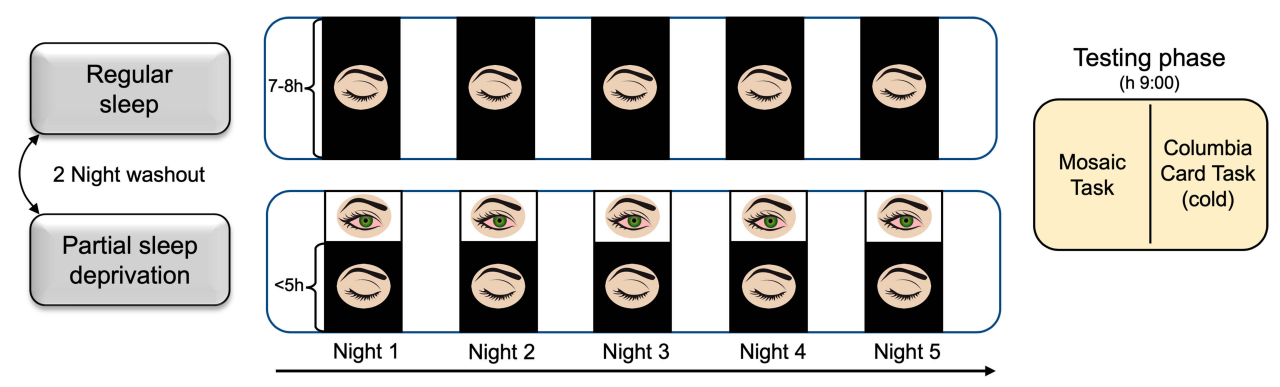

Figure 2 Experimental protocol of Experiment 2.

Notes: Schematic representation of the experimental protocol of Experiment 2, consisting of regular sleep and partial sleep deprivation condition, presented in counterbalanced order and separated by two washout nights. The Testing phase was scheduled the morning following the fifth night of each condition. 
Calculation of the sleep parameters was performed offline using a custom-written MATLAB program with a graphical user interface (version 2018a, The MathWorks, Inc., Natick, Massachusetts, USA), using the default medium threshold setting. The program was obtained directly from the authors ${ }^{58}$ and represents a validated method to transform accelerometry data into the traditional actigraphic movement counts. To obtain a subjective sleep assessment, each morning of the two experimental weeks, all the participants completed a sleep diary to report their subjective sleep duration and sleep quality.

The same three variables as in Experiment 1 (TST, EFF\%, WASO) were obtained by the Geneactiv data for the five nights of each experimental condition, with the support of sleep diaries.

\section{Testing Phase}

Experiment 2 was identical to Experiment 1 as far as the Testing phase is concerned.

\section{Data Analysis}

Similar to Experiment 1, perceived sleepiness (KSS) and negative mood index (NMI) scores have been submitted to a paired samples $t$-test, comparing the two conditions (RS, PSD), to assess the influence of the partial sleep deprivation on these subjective measures.

The same analysis was separately applied to the mean of the five nights of the two experimental conditions of the sleep diary (S-TST, S-WASO) and actigraphic variables (TST, EFF\%, WASO), to evaluate the differences in the amount/quality of sleep between the two conditions.

To evaluate the effect of the five nights of partial sleep deprivation on decision-making and on risk-taking, the same analyses adopted in Experiment 1 have been applied to the main variables of both tasks (DTD and TC for the MT and CCTc, respectively). As for Experiment 1, to better understand and isolate the effects attributable to the experimental sleep curtailment, we ran specific follow-up models described in the "Results" section. In case of significant effects, Bonferroni post hoc tests were carried out.

For all the analyses, the level of significance was always set at $\mathrm{p}<0.05$.

\section{Results}

\section{Experiment I: Total Sleep Deprivation} Actigraphic and Subjective Sleep Variables

Table 1 reports the results of the analysis of actigraphic and sleep diary variables. Both the objective and subjective measures of mean total sleep time and WASO did not differ between the five nights preceding the two experimental conditions. Moreover, the actigraphy showed no difference in sleep efficiency between the five nights before the two experimental conditions (RS, TSD).

\section{Subjective Sleepiness and Mood Variables}

The subjective sleepiness scores (KSS) were significantly different between conditions (RS: mean $\pm \mathrm{SE}, 3.06 \pm 0.27$; TSD: $\left.7.16 \pm 0.27 ; \mathrm{t}_{31}=-14.01, \mathrm{p}<0.001\right)$. Likewise, $\operatorname{mood}$ scores (NMI) were significantly different (RS: $9.18 \pm 1.00$; TSD: $\left.15.59 \pm 1.31 ; \mathrm{t}_{31}=-4.59, \mathrm{p}<0.001\right)$.

\section{Mosaic Task}

The analysis of DTD variable showed that the Condition factor $\left(\mathrm{F}_{1,539}=2.10, \mathrm{p}=0.15\right)$ and the Condition $\mathrm{x}$ Difficulty level interaction $\left(\mathrm{F}_{2,539}=1.31, \mathrm{p}=0.27\right)$ were not significant. By contrast, the Difficulty level (easy, medium, difficult) was significant $\left(\mathrm{F}_{2,539}=14.41, \mathrm{p}<0.001\right)$. Post hoc comparisons revealed that the participants drew significantly fewer tiles when the task was easy (mean $\pm \mathrm{SE}, 4.05 \pm 0.39$ ) than when it was medium $(4.71 \pm 0.39 ; \mathrm{p}=0.003)$ and difficult $(5.12 \pm$ $0.39 ; \mathrm{p}<0.001)$. Of note, the random intercept was significant (LRT $=320, p<0.001$ ), suggesting high variability in information gathering across participants. Specifically, those who drew fewer tiles exhibited a high level of reflection impulsivity, collecting a smaller amount of information before making a decision, while others showed a low level of reflection impulsivity, collecting a greater amount of information. To further investigate these differences, we assigned participants to two subgroups (High and Low Reflection

Table I Actigraphic and Subjective Sleep Parameters of Experiment I

\begin{tabular}{|l|l|l|l|l|}
\hline & RS & TSD & & \\
\cline { 2 - 5 } & Mean \pm SE & Mean \pm SE & t $_{31}$ & p \\
\hline \multicolumn{5}{|l|}{ Objective sleep variables (actigraphy) } \\
\hline TST (min) & $447.20 \pm 6.36$ & $457.51 \pm 8.29$ & -1.60 & 0.12 \\
EFF\% & $95.39 \pm 0.57$ & $94.86 \pm 0.57$ & 1.17 & 0.25 \\
WASO (min) & $16.54 \pm 2.65$ & $19.77 \pm 2.66$ & -1.37 & 0.18 \\
\hline Subjective sleep variables (diary) \\
\hline S-TST (min) & $464.74 \pm 5.53$ & $479.65 \pm 7.36$ & -0.60 & 0.55 \\
S-WASO (min) & $4.06 \pm 0.79$ & $4.96 \pm 0.90$ & -0.99 & 0.33 \\
\hline
\end{tabular}

Notes: Mean (and standard error) of objective (actigraphic) and subjective (diary) sleep parameters of the five nights preceding each condition (regular sleep, total sleep deprivation). $t$-Test analysis results ( $t$ and $p$ ) are also shown.

Abbreviations: TST, total sleep time; EFF, sleep efficiency; WASO, wake after sleep onset; S-TST, subjective total sleep time; S-WASO, subjective wake after sleep onset. 
impulsivity, respectively) based on a median split of the DTD variable in the RS condition, assumed to represent each participant's habitual performance. This categorization was added to the omnibus analysis model as a between-subject factor (Reflection impulsivity). Although the analysis controlled for the interaction between Reflection impulsivity and Difficulty level factors, these effects were not shown because not needed for discussing the effect of TSD.

As expected, the Reflection impulsivity factor was significant (High Reflection impulsivity: $3.18 \pm 0.38$; Low Reflection impulsivity: $6.07 \pm 0.38 ; \mathrm{F}_{1,30}=28.69$, $\mathrm{p}<0.001)$. Additionally, the analysis yielded a significant Reflection impulsivity $\mathrm{x}$ Condition interaction $\left(\mathrm{F}_{1,534}\right.$ $=19.76, \mathrm{p}<0.001$, see Figure 3). High and Low Reflection impulsivity subgroups were differently affected by one night of sleep deprivation. Post hoc comparisons indicated that the Low Reflection impulsivity subgroup drew significantly fewer tiles in the TSD condition compared to the RS condition $(p<0.001)$. Nevertheless, this subgroup still drew more tiles compared to the High Reflection impulsivity participants in the TSD condition $(\mathrm{p}=0.003)$. Instead, sleep deprivation did not affect the performance of the High Reflection impulsivity subgroup $(\mathrm{p}=0.23)$. The threeway interaction was not significant $\left(\mathrm{F}_{2,534}=0.98, \mathrm{p}=0.37\right)$. Control analyses indicated that all significant effects survived the covariance of mood scores (NMI).

\section{Columbia Card Task}

As shown in Table 2, the Condition effect and the interactions between Condition (RS, TSD) and task design factors (ie, Gain amount, Number of loss cards, and Loss amount) were not significant in the analysis of TC variable. As expected, Gain amount (10, 20, 30 points per card), Number of loss cards (1,2,3), and Loss amount (250, 500, 750 points) influenced participants' decisions in each trial. Post hoc comparisons revealed that participants turned more cards in the trials where the gain amount was 30 points per card (mean \pm SE, $12.88 \pm$ 0.84), compared to $10 \quad(11.92 \pm 0.84 ; \mathrm{p}=0.005)$. Participants also turned more cards in the trials in which the number of loss cards was $1(15.61 \pm 0.84)$, compared to $2(11.97 \pm 0.84 ; \mathrm{p}<0.001)$ and $3(9.73 \pm 0.84 ; \mathrm{p}<0.001)$, and when it was 2 compared to $3(\mathrm{p}<0.001)$. Additionally, the trials where the points that could be lost were 250 $(14.02 \pm 0.84)$ elicited more turned cards than trials with a higher penalty (500: $12.22 \pm 0.84 ; 750: 11.08 \pm 0.84$; all $\mathrm{p}<0.001)$.

Once again, the random intercept effect was significant (LRT=874, $\mathrm{p}<0.001$ ), suggesting high variability in risktaking across participants. Those who were more inclined to risk in the RS condition (assumed as baseline) turned more cards than those who were less risk-inclined. Accordingly, the sample was split into two subgroups, referred to as High Risk-taking and Low Risk-taking, based on the median calculated on the TC mean scores of RS condition. This Risk-taking factor was included in the omnibus model. In reporting results in Table 3, we omitted the interactions between Risk-taking and task design factors because not needed for discussing the effect of TSD.

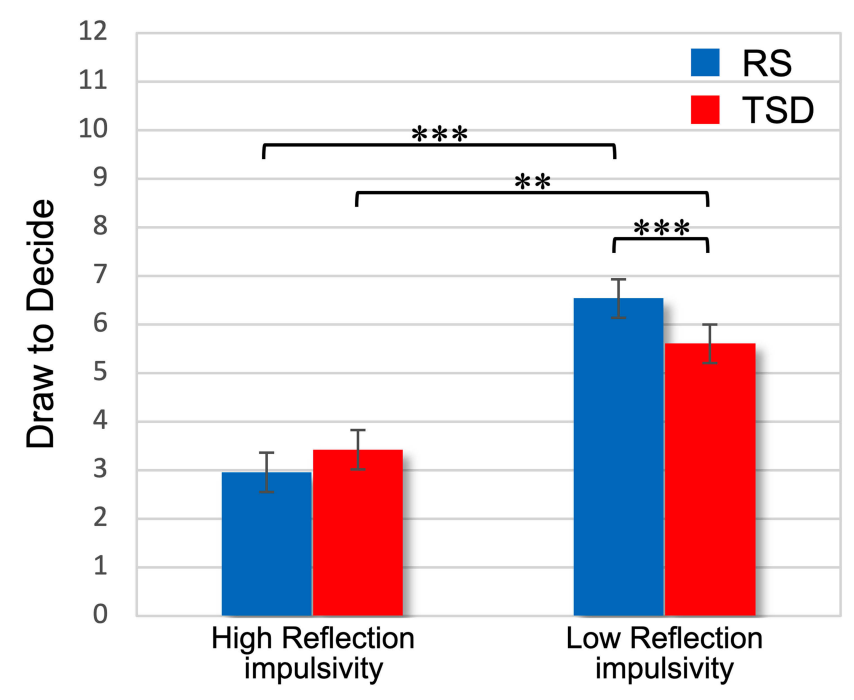

Figure 3 Reflection impulsivity $\times$ Sleep condition interaction on Mosaic Task performance in Experiment $\mathrm{I}$.

Notes: Mean (and standard error) of the trial-by-trial Draw To Decide mean scores in the two conditions (regular sleep, total sleep deprivation) for the High Reflection impulsivity and Low Reflection impulsivity subgroups. ${ }^{*} \mathrm{p}<0.01$; ${ }^{* * *} \mathrm{p}<0.001$. 
Table 2 Main Effects and Interactions of Sleep Condition and Task Design Factors for Turned Cards variable (Experiment I)

\begin{tabular}{|l|l|l|l|}
\hline & F-Test & $\begin{array}{l}\text { Degrees of } \\
\text { Freedom }\end{array}$ & P \\
\hline Condition & 0.39 & 1,1683 & 0.53 \\
Gain amount & 5.13 & 2,1683 & 0.01 \\
Number of loss cards & 192.07 & 2,1683 & $<0.001$ \\
Loss amount & 47.92 & 2,1683 & $<0.001$ \\
Condition $\times$ Gain amount & 0.24 & 2,1683 & 0.79 \\
Condition $\times$ Number of loss cards & 0.26 & 2,1683 & 0.77 \\
Condition $\times$ Loss amount & 0.24 & 2,1683 & 0.79 \\
\hline
\end{tabular}

As expected, the Risk-taking factor was significant (Low Risk-taking: $9.13 \pm 0.82$; High Risk-taking: $15.75 \pm 0.82$ ). The model indicated a significant effect for the interaction between Risk-taking and Condition, pointing to a differential effect of TSD condition between High Risk-taking and Low Risk-taking subgroups. Post hoc comparisons (Figure 4) revealed that the Low Risk-taking subgroup turned significantly more cards in the TSD condition compared to the RS condition $(p=0.02)$. Nevertheless, the Low Risk-taking subgroup was still more cautious than the High Risk-taking subgroup, even in the TSD condition $(\mathrm{p}<0.001)$. No difference for the High Risk-taking participants was obtained comparing the two experimental conditions $(\mathrm{p}=0.20)$. Additionally, none of the interactions among Condition, Risk-taking, and one of the task design factors were significant. Control analyses with NMI covariance confirmed all the significant effects.

\section{Experiment 2: Partial Sleep Deprivation} Actigraphic and Subjective Sleep Variables

Actigraphic recordings and sleep diary data confirmed the established sleep duration for the PSD condition (TST $<5$ hours), which was significantly lower than the RS condition.

Table 3 Main Effects and Interactions of Sleep Condition and Task Design Factors for Turned Cards variable, with the Inclusion of the Risk-Taking Factor (Experiment I)

\begin{tabular}{|c|c|c|c|}
\hline & F-Test & $\begin{array}{l}\text { Degrees } \\
\text { of } \\
\text { Freedom }\end{array}$ & $\mathbf{p}$ \\
\hline Risk-taking & 32.69 & 1,30 & $<0.001$ \\
\hline Condition $\times$ Risk-taking & 13.88 & 1,1670 & $<0.001$ \\
\hline Condition $\times$ Risk-taking $\times$ Gain amount & 0.13 & 2,1670 & 0.88 \\
\hline $\begin{array}{l}\text { Condition } \times \text { Risk-taking } \times \text { Number of } \\
\text { loss cards }\end{array}$ & 0.44 & 2,1670 & 0.64 \\
\hline Condition $\times$ Risk-taking $\times$ Loss amount & 0.55 & 2,1670 & 0.58 \\
\hline
\end{tabular}

Furthermore, following prolonged partial sleep deprivation, sleep efficiency increased, while WASO decreased, suggesting an increased homeostatic drive to sleep (Table 4).

\section{Subjective Sleepiness and Mood Variables}

The analysis of subjective sleep measures (KSS) showed significant differences between conditions (RS: mean \pm $\mathrm{SE}, 3.36 \pm 0.31$; PSD: $5.60 \pm 0.35 ; \mathrm{t}_{41}=-6.21, \mathrm{p}<0.001$ ), which also differed in NMI scores (RS: $13.29 \pm 1.32$; PSD: $\left.18.71 \pm 1.30 ; \mathrm{t}_{41}=-3.90, \mathrm{p}<0.001\right)$.

\section{Mosaic Task}

The Condition factor (RS: mean \pm SE, $5.53 \pm 0.55$; PSD: 5.04 $\left.\pm 0.55 ; \mathrm{F}_{1,709}=8.40, \mathrm{p}=0.004\right)$ and the difficulty level factor were significant $\left(\mathrm{F}_{2,709}=3.24, \mathrm{p}=0.04\right)$. Post hoc comparison indicated that participants drew significantly fewer tiles in the easy level of the task $(5.10 \pm 0.55)$ than in the difficult level $(5.55 \pm 0.55 ; \mathrm{p}=0.03)$. Instead, the Condition $\mathrm{x}$ Difficulty level interaction was not significant $\left(\mathrm{F}_{2,709}=0.36, \mathrm{p}=0.70\right)$.

Of note, the random intercept effect was significant (LRT=711, p<0.001). As in Experiment 1, we split the sample into two subgroups (High Reflection impulsivity, Low Reflection impulsivity), and the Reflection impulsivity factor was included in a new model. The Reflection impulsivity factor was significant (High Reflection impulsivity: $2.57 \pm 0.50$; Low Reflection impulsivity: $8.00 \pm 0.50 ; \mathrm{F}_{1,40}=64.10, \mathrm{p}<0.001$ ), as well as the Reflection impulsivity $\mathrm{x}$ Condition interaction $\left(F_{1,704}=27.64, p<0.001\right.$, see Figure 5). The five nights of partial sleep deprivation differently affected High and Low Reflection impulsivity subgroups. Post hoc comparisons revealed that the Low Reflection impulsivity subgroup drew significantly fewer tiles in the PSD condition than in the RS condition $(p<0.001)$, while the performance of participants who were higher on reflection impulsivity was not affected by the prolonged partial sleep deprivation $(\mathrm{p}=0.62)$. Again, notwithstanding this effect, participants who were lower on reflection impulsivity at baseline drew more tiles than the other subgroup even in the PSD condition $(\mathrm{p}<0.001)$. The three-way interaction was not significant $\left(\mathrm{F}_{2,704}=0.36, \mathrm{p}=0.70\right)$. Of note, adding the NMI covariate did not affect the significant effects obtained in the models.

\section{Columbia Card Task}

As shown in Table 5, the Condition factor was significant (RS: mean \pm SE, $11.14 \pm 0.61$; PSD: $11.95 \pm 0.61$ ). Moreover, the analysis showed that Gain amount, Number of loss cards, and Loss amount affected the 


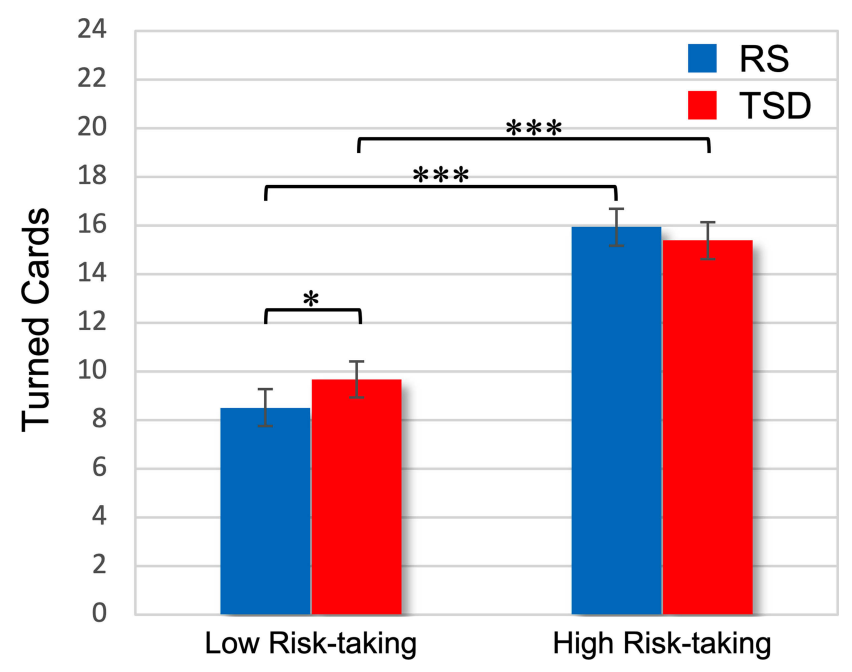

Figure 4 Risk-taking $\times$ Sleep condition interaction on Columbia Card Task (cold) performance in Experiment $\mathrm{I}$.

Notes: Mean (and standard error) of the trial-by-trial Turned Cards mean scores in the two conditions (regular sleep, total sleep deprivation) for the Low Risk-taking and High Risk-taking subgroups. ${ }^{*} \mathrm{p}<0.05 ;{ }^{* * *} \mathrm{p}<0.001$.

number of turned cards. Participants turned fewer cards in the trials in which the gain amount was 10 points per card (10.56 \pm 0.62$)$, compared to $20(11.65 \pm 0.62 ; \mathrm{p}=0.003)$ or 30 points per card $(12.42 \pm 0.62 ; \mathrm{p}<0.001)$. Participants turned more cards in the trials in which the number of loss cards was 1 (14.84 \pm 0.62$)$, compared to levels $2(11.08 \pm$ $0.62 ; \mathrm{p}<0.001)$ and $3(8.71 \pm 0.62 ; \mathrm{p}<0.001)$, and when it was 2 , compared to level 3 ( $\mathrm{p}<0.001)$. Additionally, participants turned more cards in the trials in which the loss amount was 250 points $(13.05 \pm 0.62)$, compared to 500 $(11.16 \pm 0.62 ; \mathrm{p}<0.001)$ and 750 points $(10.43 \pm 0.62$; $\mathrm{p}<001)$. On the other hand, the interactions between the Condition and the task design factors were not significant.

Table 4 Actigraphic and Subjective Sleep Parameters of Experiment 2

\begin{tabular}{|c|c|c|c|c|}
\hline & RS & PSD & \multirow[t]{2}{*}{$\mathbf{t}_{41}$} & \multirow[t]{2}{*}{$\mathbf{p}$} \\
\hline & Mean \pm SE & Mean \pm SE & & \\
\hline \multicolumn{5}{|c|}{ Objective sleep variables (actigraphy) } \\
\hline TST (min) & $417.86 \pm 4.77$ & $263.84 \pm 2.50$ & 35.83 & $<0.001$ \\
\hline EFF\% & $89.20 \pm 0.65$ & $90.37 \pm 0.49$ & -2.08 & 0.04 \\
\hline WASO (min) & $29.90 \pm 1.88$ & $15.09 \pm 0.92$ & 11.84 & $<0.001$ \\
\hline \multicolumn{5}{|c|}{ Subjective sleep variables (diary) } \\
\hline S-TST (min) & $454.71 \pm 5.02$ & $289.76 \pm 1.49$ & 36.89 & $<0.001$ \\
\hline S-WASO (min) & $4.03 \pm 0.70$ & $2.33 \pm 0.59$ & 2.04 & 0.05 \\
\hline
\end{tabular}

Notes: Mean (and standard error) of objective (actigraphic) and subjective (diary) sleep parameters of the two conditions (regular sleep, partial sleep deprivation). $t$-Test analysis results ( $t$ and $\mathrm{p}$ ) are also shown.

Abbreviations: TST, total sleep time; EFF, sleep efficiency; WASO, wake after sleep onset; S-TST, subjective total sleep time; S-WASO, subjective wake after sleep onset.
Instead, the random intercept effect was significant (LRT=551, $\mathrm{p}<0.001$ ), showing a wide variability among participant responses. As in Experiment 1, we split the sample into two subgroups (Low Risk-taking, High Risktaking), and the Risk-taking factor was included in the model. The results are reported in Table 6.

The Risk-taking factor was significant (Low Risk-taking: $8.73 \pm 0.57$; High Risk-taking: $14.36 \pm 0.57$ ), as well as the Risk-taking $\mathrm{x}$ Condition interaction. The High Risk-taking and Low Risk-taking subgroups were differently affected by the five nights of partial sleep deprivation (see Figure 6). Post hoc comparisons showed that under PSD, the Low Risktaking subgroup turned significantly more cards than in the RS condition $(\mathrm{p}<0.001)$, while the High Risk-taking participants turned the same number of cards in both conditions $(p=0.67)$. Again, notwithstanding this effect, the two subgroups continued to show a different risk-taking behavior also in the PSD condition ( $\mathrm{p}<0.001)$. None of the interactions among Condition, Risk-taking, and one of the task factors were significant. Control analyses confirmed that all significant effects survived the NMI covariance.

\section{Discussion}

In this study, we showed that five consecutive nights of partial sleep deprivation reduced data gathering before making a decision and increased risk propensity in deliberative decision-making. By contrast, one night of total sleep deprivation was not enough to affect data gathering and risk-taking behavior. These findings suggested that prolonged sleep curtailment is more effective than one night of sleep deprivation 


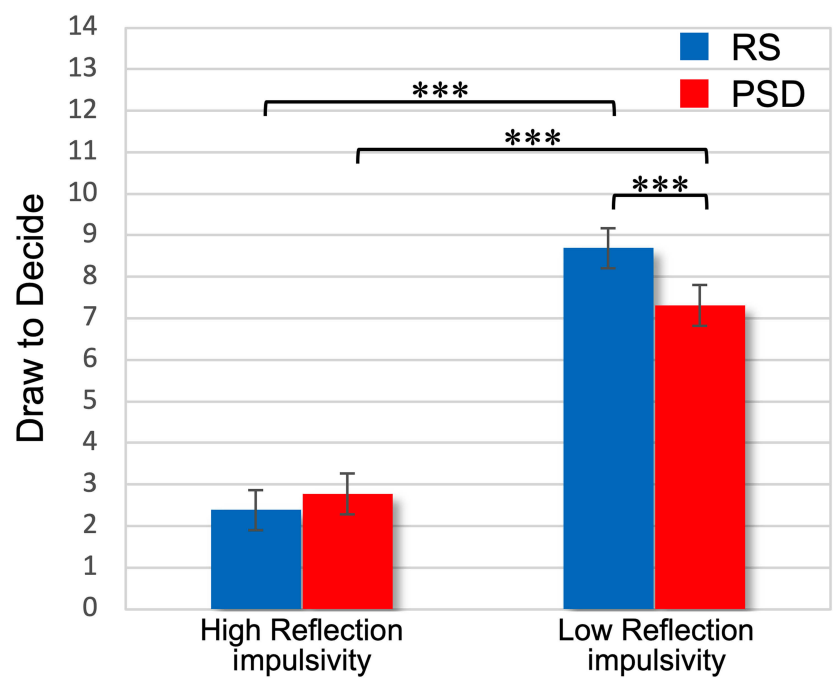

Figure 5 Reflection impulsivity $\times$ Sleep condition interaction on Mosaic Task performance in Experiment 2.

Notes: Mean (and standard error) of the trial-by-trial Draw To Decide mean scores in the two conditions (regular sleep, partial sleep deprivation) for the High Reflection impulsivity and Low Reflection impulsivity subgroups. ${ }^{* * *} \mathrm{p}<0.001$.

in modifying the specific decision-making behaviors implied in the two tasks used in the present study. This result is in line with previous research, which suggested that PSD potentially has a more detrimental effect on cognitive performance than TSD. $^{59}$ This finding may also have practical implications, considering that an inadequate amount of sleep is a widespread condition in our modern western societies.

Of note, in both the experiments, we evidenced a wide inter-individual variability in the participants' responses on both tasks. In particular, following one night of total sleep deprivation, subjects tended to differentiate according to their habitual performance rather than to experimental manipulations. This result led us to hypothesize that there could be individual difference factors underlying decisionmaking processes that could not be altered by the experimental manipulations. After all, both MT and CCTc have been created and are currently used as tests of individual

Table 5 Main Effects and Interactions of Sleep Condition and Task Design Factors for Turned Cards variable (Experiment 2)

\begin{tabular}{|l|l|l|l|}
\hline & F-Test & $\begin{array}{l}\text { Degrees of } \\
\text { Freedom }\end{array}$ & P \\
\hline Condition & 9.15 & 1,2213 & 0.003 \\
Gain amount & 16.48 & 2,2213 & $<0.001$ \\
Number of loss cards & 181.89 & 2,2213 & $<0.001$ \\
Loss amount & 34.71 & 2,2213 & $<0.001$ \\
Condition $\times$ Gain amount & 0.29 & 2,2213 & 0.75 \\
Condition $\times$ Number of loss cards & 0.89 & 2,2213 & 0.41 \\
Condition $\times$ Loss amount & 0.17 & 2,2213 & 0.85 \\
\hline
\end{tabular}

differences in probabilistic reasoning and risk-taking in deliberative decision-making. ${ }^{22,35}$ To aid in the interpretation, we carried out some exploratory analyses, assuming a differential effect of both the experimental sleep manipulations depending on the participants' habitual (baseline) decision-making behavior. The analyses showed that both total and prolonged partial sleep deprivation reduced willingness to gather information before deciding for participants who behaved more reflectively in the MT. Sleep loss also caused an increment in risk appetite specifically in less risk-inclined subjects in the CCTc.

Of note, the median split procedure applied to the data of the two tasks gave rise to subgroups which were not fully overlapping in both the experiments, since participants concomitantly assigned to High Reflection impulsivity and High Risk-taking subgroups were $65.63 \%$ in Experiment 1, and 52.38\% in Experiment 2. This outcome confirmed that different decision-making constructs subtended and were assessed by the two tasks. ${ }^{22}$ This is not surprising considering the specific performance required by each task, with MT requiring data gathering in a context of probabilistic (Bayesian) reasoning, and the $\mathrm{CCTc}$ reflecting a risk-taking process. Accordingly, herein we separately discuss the results for each task.

\section{Effect of TSD and PSD on Reflection Impulsivity}

If we consider the whole sample, one night of total sleep deprivation did not influence MT performance. On the 
Table 6 Main Effects and Interactions of Sleep Condition and Task Design Factors for Turned Cards variable, with the Inclusion of the Risk-Taking Factor (Experiment 2)

\begin{tabular}{|c|c|c|c|}
\hline & F-Test & $\begin{array}{l}\text { Degrees of } \\
\text { Freedom }\end{array}$ & $\mathbf{p}$ \\
\hline Risk-taking & 48.24 & 1,40 & $<0.001$ \\
\hline Condition $\times$ Risk-taking & 29.01 & 1,2200 & $<0.001$ \\
\hline Condition $\times$ Risk-taking $\times$ Gain amount & 0.24 & 2,2200 & 0.79 \\
\hline $\begin{array}{l}\text { Condition } \times \text { Risk-taking } \times \text { Number of } \\
\text { loss cards }\end{array}$ & 0.17 & 2,2200 & 0.84 \\
\hline Condition $\times$ Risk-taking $\times$ Loss amount & 1.91 & 2,2200 & 0.15 \\
\hline
\end{tabular}

other hand, five nights of sleep restriction lead to an overall reduction in data gathering before making decisions. Nevertheless, follow up analyses that took into account the participant's habitual performance showed consistent results between the two experiments. Participants characterized by a greater data gathering in the RS condition before making a decision (Low Reflection impulsivity subgroups) based their responses on a significantly smaller amount of information following both conditions of sleep deprivation (TSD, PSD). On the other hand, the performance of participants who drew fewer tiles in the RS condition (High Reflection impulsivity subgroups) did not seem to be affected by both TSD and PSD manipulation. To account for these peculiar results, we refer to a substantial literature supporting the existence of two interactive processes underlying reasoning, and putatively involved in MT: the "intuitive" and the "analytic" processing. ${ }^{60-63}$ The former involves rapid, automatic, and effortless processing, while the latter purely deliberative and effortful processing. In this view, the largest published Beads task study to date ${ }^{64}$ showed that the analytic cognitive style specifically predicted performance in this task in a large non-clinical sample. Additionally, Brosnan et $\mathrm{al}^{65}$ showed a link between evidence accumulation in the Beads task and performance in an analytic reasoning task. Accordingly, we hypothesized that the reduction in the number of drawn tiles following both total and (cumulative) partial sleep deprivation could reflect an impairment of the effortful analytic processing, in favor of the automatic, intuitive one. Sleep loss, impacting preferentially the purely deliberative reasoning process, could lead to react more impulsively in response to the arrival of new information in an effortful probabilistic reasoning task like the MT. Accordingly, participants putatively characterized by an under-utilization of the analytic processing in the RS condition (High Reflection impulsivity subgroups) were not affected by the experimental sleep manipulations.

In support of this interpretation, we could refer to the well-known adverse consequences of an inadequate amount of sleep on working memory (WM) function. ${ }^{66-69}$ Since the analytic processing heavily burdens the WM system, an impaired WM could mediate the decrease in drawn tiles following sleep loss. Alterations in the ability to maintain and manipulate increasing information following sleep deprivation could imply that individuals were less inclined to evaluate the probability of a given outcome carefully, ${ }^{17}$ leading to an approximation of their responses. ${ }^{70}$ Coherently, the

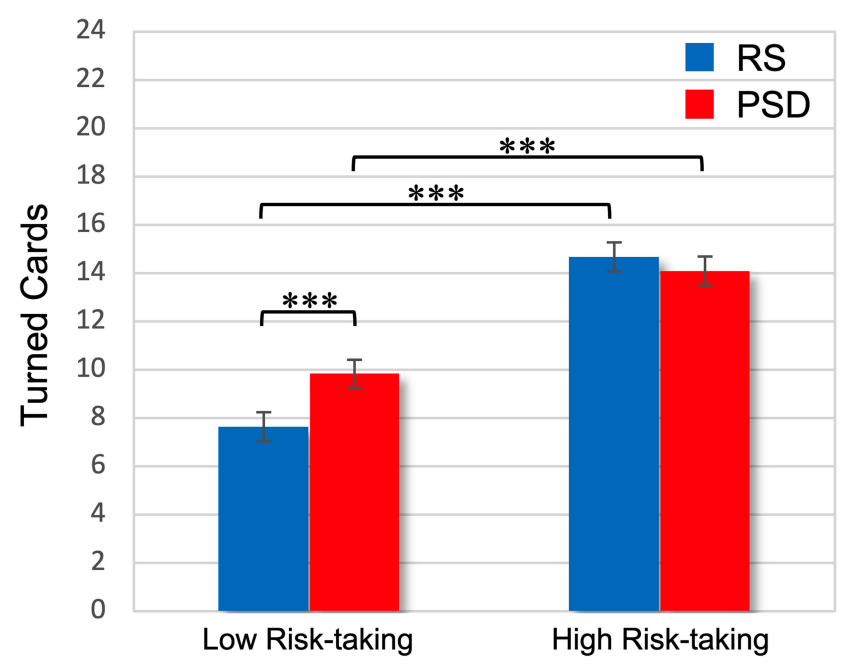

Figure 6 Risk-taking $\times$ Sleep condition interaction on Columbia Card Task (cold) performance in Experiment 2.

Notes: Mean (and standard error) of the trial-by-trial Turned Cards mean scores in the two conditions (regular sleep, partial sleep deprivation) for the Low Risk-taking and High Risk-taking subgroups. $* * * p<0.001$. 
current literature on the Beads task widely proposed a link between limited information gathering (jumping to conclusion bias) and deficits of the WM system. ${ }^{71-73}$

Remarkably, a differential effect depending on baseline performance in the Beads task has been already shown in a study aimed to evaluate the consequence of methylphenidate (MPH) intake. ${ }^{74}$ In this study, the authors showed that, after a similar median split of the sample based on placebo condition performance, MPH led to a decrement in evidence collected in the subgroup which gathered more information in the baseline performance (equivalent to our Low Reflection impulsivity subgroups). This similarity is interesting, considering that both MPH and sleep deprivation have been linked to an altered dopamine signaling in the striatum, ${ }^{75-77}$ which is thought to be implied in Beads task performance. $^{73,78}$ Further investigations may clarify the putative role of the dopaminergic system to explain this specific pattern of results. Finally, the significant effect obtained in the entire sample of Experiment 2 was not consistent with a recent prolonged sleep restriction study using the Beads task. ${ }^{79}$ However, in Reeve et al ${ }^{79}$ sleep was restricted to 5 hours for only three consecutive nights, and the easiest version of the task (85:15) was adopted. These substantial differences could explain the discrepant results between studies.

\section{Effect of TSD and PSD on Risk-Taking}

Unlike total sleep deprivation, prolonged partial sleep deprivation significantly affected the CCTc performance, leading to an increment in risk-taking behavior. Additionally, follow up analyses showed that both sleep manipulations (TSD and PSD) increased risk-taking, but only in participants who showed a more cautious behavior when they were well-rested. These results are consistent with previous studies which have shown that, if there could be an effect of sleep deprivation on gambling tasks, this would be in the direction of greater risk seeking. ${ }^{33,34} \mathrm{In}$ particular, fMRI studies have shown that sleep deprivation resulted in functional alterations of brain areas involved in win/loss signal processing. ${ }^{80}$ These results have been interpreted as an increase of the expectation of winning and an attenuation of the loss aversion, ${ }^{80}$ linked to a behavioral shift from preventing losses to pursuing gains following sleep deprivation. ${ }^{8}$ A putative mechanism involved in the well-known increment of risk-taking propensity induced by sleep loss is the impairment of the inhibitory systems, which lead to impulsive behavior to pursuing gains. ${ }^{34}$ On the other hand, total and partial sleep deprivation did not seem to affect the performance of the subgroups showing a higher risk-inclined behavior in the baseline condition (High Risktaking subgroups). Conceiving the CCTc performance as a function of dispositional factors, Buelow ${ }^{81}$ showed a relation between low-risk propensity in this task and a higher behavioral inhibition measure, involving greater sensitivity to punishment signals. Additionally, also the impulsivity construct seems to be implied in the CCTc performance. Indeed, although this task was designed to assess deliberative decision processes, higher impulsivity involves poor planning skills and inappropriate responses, impairing the ability to consider the different decision options carefully. ${ }^{82}$ In this view, the impulsivity trait predicted a higher amount of turned cards in CCTc. ${ }^{82}$

Based on these separate lines of evidence, we hypothesize that the increment in risk-taking of habitually risk-averse individuals could reflect an impairment of the behavioral inhibition systems, which triggered more risky choices. On the other hand, participants more inclined to riskseeking could be not influenced by the sleep manipulations because they might be already dispositionally deficient in inhibitory processes, and this situation could have hindered the emergence of a specific effect of sleep deprivation in this group.

Finally, similar to MT, another hypothetical interpretation of our pattern of results involves a sleep loss-induced impairment of WM, since CCTc typically requires deliberative elaboration implicating WM. ${ }^{32}$ In this view, optimal WM function has been linked to both lower risk-taking behavior $^{81}$ and greater information use ${ }^{31}$ in CCTc. Therefore, a WM dysfunction could explain the increase in risk propensity specifically showed by the sub-sample who approached more cautiously to the task.

\section{Limitations}

It should be acknowledged that the composition of the experimental sample consisted of healthy young good sleepers. This feature could limit the generalization of our results to the whole population. Another potential limitation of our findings concerns the limited duration of the washout period (2 nights) in Experiment 2. Nevertheless, this unlikely influenced the results, given that the presentation order of conditions was counterbalanced, and control analyses (not reported) failed to show significant differences between the two different presentation orders. A final consideration regards a typical characteristic of the prolonged sleep restriction protocols, ie, the absence of a laboratory setting. This situation in Experiment 2 implied limited 
experimental control on possible confounding environmental factors.

\section{Conclusion}

The results of our study showed that an inadequate amount of sleep influences reflection impulsivity and risktaking depending on the habitual individual way of responding during deliberative decision-making. Our findings support the assumption that the consequences of sleep loss could be related to individual differences. Interindividual differences in the sleep deprivation effect have already been documented in the decision-making literature. For example, a gender-specific effect of sleep loss has been demonstrated both in impulsivity and economic decision tasks. ${ }^{9,83}$ Furthermore, in an economic decision task, sleep deprivation has been linked to an increment in risk-taking selectively in individuals with high reflection trait scores, configuring a specific effect on dispositionally less impulsive participants. ${ }^{83}$ Additionally, concerning reward/punishment processing, a recent study ${ }^{84}$ revealed a relation between the consequences of sleep deprivation and a particular genetic polymorphism involved in synaptic dopamine regulation. On the other hand, many studies on reward-related decision-making have failed to show univocal behavioral or functional differences induced by sleep deprivation within the examined samples. ${ }^{85-87}$

The existence of possible interactions between dispositional factors and the effects of sleep deprivation could provide an interpretative key to account for the inconsistencies in this field. Accordingly, our results support the hypothesis that individual differences may hide grouplevel significance and produce misleading conclusions in sleep deprivation protocols. ${ }^{4}$ For these reasons, we caution that further investigation is warranted on this matter. Future sleep deprivation studies could explicitly consider individual differences implied in the decision-making task adopted, using a detailed preliminary screening. This strategy could help to support the hypothesis of a putative interaction between individual differences and the influence of sleep loss on decision performance.

In conclusion, our findings suggest that, under the effects of total and partial sleep loss, people habitually more reflective tend to gather less evidence to make a decision, and more cautious individuals become more prone to risk-taking in situations requiring decisionmaking based on deliberative reasoning.

\section{Acknowledgments}

We thank Giulia Amicucci, Elisa Asisani, Jasmin Cascioli, Irene D'Avino, Eleonora Grandi and Lorenzo Viselli for their help in data collection.

\section{Funding}

This research did not receive any specific grant from funding agencies in the public, commercial, or not-forprofit sectors.

\section{Disclosure}

The authors report no conflicts of interest in this work.

\section{References}

1. Sleep and sleep disorders. Centers for disease, control and prevention; 2015. Available from: https://www.cdc.gov/sleep/index. $\mathrm{html}$. Accessed May 11, 2020.

2. Walker MP. Why We Sleep. London, UK: Penguin; 2017.

3. Dinges DF, Pack F, Williams K, et al. Cumulative sleepiness, mood disturbance, and psychomotor vigilance performance decrements during a week of sleep restricted to 4-5 hours per night. Sleep. 1997;20 (4):267-277.

4. Krause AJ, Simon EB, Mander BA, et al. The sleep-deprived human brain. Nat Rev Neurosci. 2017;18(7):404-418. doi:10.1038/nrn.2017.55

5. Harrison Y, Horne JA. The impact of sleep deprivation on decision making: a review. $J$ Exp Psychol Appl. 2000;6(3):236-249. doi:10.1037//1076-898X.6.3.236

6. Killgore WD, Balkin TJ, Wesensten NJ. Impaired decision making following 49 h of sleep deprivation. J Sleep Res. 2006;15(1):7-13. doi:10.1111/j.1365-2869.2006.00487.x

7. McKenna BS, Dickinson DL, Orff HJ, Drummond SPA. The effects of one night of sleep deprivation on known-risk and ambiguous-risk decisions. J Sleep Res. 2007;16(3):245-252. doi:10.1111/j.13652869.2007.00591.x

8. Venkatraman V, Huettel SA, Chuah LY, Payne JW, Chee MWL. Sleep deprivation biases the neural mechanisms underlying economic preferences. J Neurosci. 2011;31(10):3712-3718. doi:10.1523/ JNEUROSCI.4407-10.2011

9. Acheson A, Richards JB, de Wit H. Effects of sleep deprivation on impulsive behaviors in men and women. Physiol Behav. 2007;91 (5):579-587. doi:10.1016/j.physbeh.2007.03.020

10. Killgore WD. Effects of sleep deprivation on morningness-eveningness traits on risk-taking. Psychol Rep. 2007;100(2):613-626. doi:10.2466/ pr0.100.2.613-626

11. Yang H, Carmon Z, Kahn B, et al. The hot-cold decision triangle: a framework for healthier choices. Mark Lett. 2012;23:457-472. doi:10.1007/s11002-012-9179-0

12. Damasio AR. Descartes' Error: Emotion, Reason, and the Human Brain. New York: Putnam; 1994.

13. Shafir E, Simonson I, Tversky A. Reason-based choice. Cognition. 1993;49(1-2):11-36. doi:10.1016/0010-0277(93)90034

14. Seguin JR, Arseneault L, Tremblay RE. The contribution of "cool" and "hot" components of decision-making in adolescence: implications for developmental psychopathology. Cogn Dev. 2007;22 (4):530-543. doi:10.1016/j.cogdev.2007.08.006

15. Jackson ML, Gunzelmann G, Whitney P, et al. Deconstructing and reconstructing cognitive performance in sleep deprivation. Sleep Med Rev. 2013;17(3):215-225. doi:10.1016/j.smrv.2012.06.007

16. Bechara A. Iowa Gambling Task Professional Manual. Lutz, FL: Psychological Assessment Resources; 2008. 
17. Dickinson DL, Drummond SPA. The effects of total sleep deprivation on Bayesian updating. Judgm Decis Mak. 2008;3:181-190.

18. Anderson C, Dickinson DL. Bargaining and trust: the effects of 36-h total sleep deprivation on socially interactive decisions. J Sleep Res. 2010;19:54-63. doi:10.1111/j.1365-2869.2009.00 767.x

19. Dickinson DL, McElroy T. Sleep restriction and circadian effects on social decisions. Eur Econ Rev. 2017;97:57-71. doi:10.1016/j. euroecorev.2017.05.002

20. Güroğlu B, van den Bos W, Rombouts SA, Crone EA. Unfair? It depends: neural correlates of fairness in social context. Soc Cogn Affect Neurosci. 2010;5(4):414-423. doi:10.1093/scan/nsq013

21. Chang LJ, Sanfey AG. Great expectations: neural computations underlying the use of social norms in decision-making. Soc Cogn Affect Neurosci. 2013;8(3):277-284. doi:10.1093/scan/nsr094

22. Lauriola M, Weller J. Personality and risk: beyond daredevils-risk taking from a temperament perspective. In: Raue M, Lermer E, Streicher B, editors. Psychological Perspectives on Risk and Risk Analysis: Theory, Models, and Applications. Cham: Springer; 2018:3-36. doi:10.1007/978-3-319-92478-6_1

23. Levin I, Xue G, Weller J, Reimann M, Lauriola M, Bechara A. A neuropsychological approach to understanding risk-taking for potential gains and losses. Front Neurosci. 2012;6:15. doi:10.3389/ fnins.2012.00015

24. Tucker AM, Whitney P, Belenky G, Hinson JM, Van Dongen HP. Effects of sleep deprivation on dissociated components of executive functioning. Sleep. 2010;33(1):47-57. doi:10.1093/sleep/33.1.47

25. Whitney P, Hinson JM. Measurement of cognition in studies of sleep deprivation. Prog Brain Res. 2010;185:37-48. doi:10.1016/B9780-444-53702-7.00003-8

26. Huq SF, Garety PA, Hemsley DR. Probabilistic judgements in deluded and non-deluded subjects. $Q J$ Exp Psychol A. 1988;40 (4):801-812. doi:10.1080/14640748808402300

27. Averbeck BB, Evans S, Chouhan V, Bristow E, Shergill SS. Probabilistic learning and inference in schizophrenia. Schizophr Res. 2011;127(1-3):115-122. doi:10.1016/j.schres.2010.08.009

28. Djamshidian A, O'Sullivan SS, Sanotsky Y, et al. Decision making, impulsivity, and addictions: do parkinson's disease patients jump to conclusions? Mov Disord. 2012;27(9):1137-1145. doi:10.1002/ mds. 25105

29. Ross RM, McKay R, Coltheart M, Langdon R. Jumping to conclusions about the beads task? A meta-analysis of delusional ideation and data-gathering. Schizophr Bull. 2015;41(5):1183-1191. doi:10.1093/ schbul/sbu187

30. Banca P, Lange I, Worbe Y, et al. Reflection impulsivity in binge drinking: behavioural and volumetric correlates. Addict Biol. 2016;21 (2):504-515. doi:10.1111/adb.12227

31. Figner B, MacKinlay R, Wilkenin F, Weber EU. Affective and deliberative processes in risky choice: age differences in risk taking in the Columbia card task. J Exp Psychol Learn Mem Cogn. 2009;35 (3):709-730. doi:10.1037/a0014983

32. Markiewicz L, Kubińska E. Information use differences in hot and cold risk processing: when does information about probability count in the Columbia card task? Front Psychol. 2015;6:1727. doi:10.3389/ fpsyg.2015.01727

33. Womack SD, Hook JN, Reyna SH, Ramos M. Sleep loss and risk-taking behavior: a review of the literature. Behav Sleep Med. 2013;11(5):343-359. doi:10.1080/15402002.2012.703628

34. Killgore WD. Sleep deprivation and behavioral risk-taking. In: Watson RR, editor. Modulation of Sleep by Obesity, Diabetes, Age, and Diet. London, UK: Academic Press; 2015:279-287. doi:10.1016/ B978-0-12-420168-2.00030-2

35. Panno A, Lauriola M, Figner B. Emotion regulation and risk taking: predicting risky choice in deliberative decision making. Cogn Emot. 2013;27(2):326-334. doi:10.1080/02699931.2012.707642
36. Lowe CJ, Safati A, Hall PA. The neurocognitive consequences of sleep restriction: a meta-analytic review. Neurosci Biobehav Rev. 2017;80:586-604. doi:10.1016/j.neubiorev.2017.07.010

37. Reynolds AC, Banks S. Total sleep deprivation, chronic sleep restriction and sleep disruption. Prog Brain Res. 2010;185:91-103. doi:10.1016/B978-0-444-53702-7.00006-3

38. Buysse DJ, Reynolds CF, Monk TH, Berman SR, Kupfer DJ. The pittsburgh sleep quality index: a new instrument for psychiatric practice and research. Psychiatry Res. 1989;28(2):193-213. doi:10.1016/0165-1781(89)90047-4

39. Curcio G, Tempesta D, Scarlata S, et al. Validity of the Italian version of the pittsburgh sleep quality index (PSQI). Neurol Sci. 2013;34 (4):511-519. doi:10.1007/s10072-012-1085-y

40. Bastien $\mathrm{CH}$, Vallières A, Morin CM. Validation of the insomnia severity index as an outcome measure for insomnia research. Sleep Med. 2011;2(4):297-307. doi:10.1016/s1389-9457(00)00065-4

41. Castronovo V, Galbiati A, Marelli S, et al. Validation study of the Italian version of the insomnia severity index (ISI). Neurol Sci. 2016;37(9):1517-1524. doi:10.1007/s10072-016-2620-z

42. Ghisi M, Flebus G, Montano A, Sanavio E, Sica C. Beck Depression Inventory-II: Edizione Italiana. Firenze, IT: Giunti Editore; 2006.

43. Moroni L, Bettinardi O, Vidotto G, et al. Anxiety and depression short scale: norms for its use in rehabilitation. Monaldi Arch Chest Dis. 2006;66:255-263. doi:10.4081/monaldi.2006.516

44. Akerstedt T, Gillberg M. Subjective and objective sleepiness in the active individual. Int $J$ Neurosci. 1990;52(1-2):29-37. doi:10.3109/ 00207459008994241

45. Stern RA, Arruda JE, Hooper CR, Wolfner GD, Morey CE. Visual analogue mood scales to measure internal mood state in neurologically impaired patients: description and initial validity evidence. Aphasiol. 1997;11:59-71. doi:10.1080/02687039708248455

46. Clark L, Robbins TW, Ersche KD, Sahakian BJ. Reflection impulsivity in current and former substance users. Biol Psychiatry. 2006;60 (5):515-522. doi:10.1016/j.biopsych.2005.11.007

47. Dalley JW, Everitt BJ, Robbins TW. Impulsivity, compulsivity, and top-down cognitive control. Neuron. 2011;69(4):680-694. doi:10.1016/j.neuron.2011.01.020

48. Djamshidian A, Sanotsky Y, Matviyenko Y, et al. Increased reflection impulsivity in patients with ephedrone-induced parkinsonism. Addiction. 2013;108(4):771-779. doi:10.1111/add.12080

49. Voon V, Irvine MA, Derbyshire K. Measuring "waiting" impulsivity in substance addictions and binge eating disorder in a novel analogue of rodent serial reaction time task. Biol Psychiatry. 2014;75 (2):148-155. doi:10.1016/j.biopsych.2013.05.013

50. Garety PA, Freeman D, Jolley S, et al. Reasoning, emotions, and delusional conviction in psychosis. J Abnorm Psychol. 2005;114 (3):373-384. doi:10.1037/0021-843X.114.3.373

51. Jacoby RJ, Abramowitz JS, Buck BE, Fabricant LE. How is the beads task related to intolerance of uncertainty in anxiety disorders? $J$ Anxiety Disord. 2014;28(6):495-503. doi:10.1016/j.janxdis.2014.05.005

52. Jacoby RJ, Abramowitz JS, Reuman L, Blakey SM. Enhancing the ecological validity of the beads task as a behavioral measure of intolerance of uncertainty. $J$ Anxiety Disord. 2016;41:43-49. doi:10.1016/j.janxdis.2016.02.003

53. Figner B, Weber EU. Who takes risks when and why? Determinants of risk taking. Curr Dir Psychol Sci. 2011;20(4):211-216. doi: $10.1177 / 0963721411415790$

54. Holper L, Murphy RO. Hemodynamic and affective correlates assessed during performance on the Columbia Card Task (CCT). Brain Imaging Behav. 2014;8(4):517-530. doi:10.1007/s11682-013-9265-9

55. Bates D, Mächler M, Bolker B, Walker S. Fitting linear mixed-effects models using lme4. J Stat Softw. 2015;67(1):1-48. doi:10.18637/jss.v067. i01

56. $\mathrm{R}$ Core Team. R: A Language and Environment for Statistical Computing (3.5.1). Computer Software. Vienna, Austria: R Foundation for Statistical Computing; 2018. 
57. Luke SG. Evaluating significance in linear mixed-effects models in R. Behav Res Methods. 2017;49(4):1494-1502. doi:10.3758/s13428016-0809-y

58. Te Lindert BHW, Van Someren EJW. Sleep estimates using microelectromechanical systems (MEMS). Sleep. 2013;36(5):781-789. doi:10.5665/sleep. 2648.7

59. Pilcher JJ, Huffcutt AI. Effects of sleep deprivation on performance: a meta-analysis. Sleep. 1996;19(4):318-326. doi:10.1093/sleep/19.4.318

60. Evans JSBT. Thinking Twice: Two Minds in One Brain. Oxford, UK: Oxford University Press; 2010.

61. Kahneman D. Thinking, Fast and Slow. New York, NY: Farrar, Straus and Giroux; 2011.

62. Stanovich KE. Rationality and the Reflective Mind. Oxford: Oxford University Press; 2011.

63. Evans JSBT, Stanovich KE. Dual-process theories of higher cognition: advancing the debate. Perspect Psychol Sci. 2013;8(3):223-241. doi:10.1177/1745691612460685

64. Ross RM, Pennycook G, McKay R, Gervais WM, Langdon R, Coltheart M. Analytic cognitive style, not delusional ideation, predicts data gathering in a large beads task study. Cogn Neuropsychiatry. 2016;21(4):300-314. doi:10.1080/13546805.2016.1192025

65. Brosnan M, Hollinworth M, Antoniadou K, Lewton M. Is empathizing intuitive and systemizing deliberative? Pers Individ Differ. 2014;66:39-43. doi:10.1016/j.paid

66. Smith ME, McEvoy LK, Gevins A. The impact of moderate sleep loss on neurophysiological signals during working-memory task performance. Sleep. 2002;25:784-794. doi:10.1093/sleep/25.7.56

67. Chee MWL, Chuah YML, Venkatraman V, Chan WY, Philip P, Dinges D. Functional imaging of working memory following normal sleep and after 24 and $35 \mathrm{~h}$ of sleep deprivation: correlations of fronto-parietal activation with performance. NeuroImage. 2006;31:419-428. doi:10.1016/j.neuroimage.2005.12.001

68. Frenda SJ, Fenn KM. Sleep less, think worse: the effect of sleep deprivation on working memory. JARMAC. 2016;5(4):463-469. doi:10.1016/j.jarmac.2016.10.001

69. Gosselin D, De Koninck J, Campbell K. Novel measures to assess the effects of partial sleep deprivation on sensory, working, and permanent memory. Front Psychol. 2017;8:1607. doi:10.3389/fpsyg.2017.01607

70. Drummond SPA, Brown GG, Stricker JL, Buxton RB, Wong EC, Gillin JC. Sleep deprivation-induced reduction in cortical functional response to serial subtraction. Neuroreport. 1999;10:3745-3748. doi:10.1097/00001756-199912160-00004

71. Garety P, Joyce E, Jolley S, et al. Neuropsychological functioning and jumping to conclusions in delusions. Schizophr Res. 2013;150 (2-3):570-574. doi:10.1016/j.schres.2013.08.035

72. Krężołek M, Pionke R, Banaszak B, Kokoszka A, Gawęda Ł. The relationship between jumping to conclusions and neuropsychological functioning in schizophrenia. Psychiatry Res. 2019;273:443-449. doi:10.1016/j.psychres.2019.01.035

73. Esslinger C, Braun U, Schirmbeck F, et al. Activation of midbrain and ventral striatal regions implicates salience processing during a modified beads task. PLoS One. 2013;8(3):e58536. doi:10.1371/ journal.pone.0058536
74. Voon V, Chang-Webb YC, Morris LS, et al. Waiting impulsivity: the influence of acute methylphenidate and feedback. Int J Neuropsychopharmacol. 2016;19(1):pyv074. doi:10.1093/ijnp/ pyv074

75. Volkow ND, Wang G, Fowler JS, et al. Therapeutic doses of oral methylphenidate significantly increase extracellular dopamine in the human brain. J Neurosci. 2001;21(2):RC121. doi:10.1523/ JNEUROSCI.21-02-j0001.2001

76. Volkow ND, Tomasi D, Wang GJ, et al. Evidence that sleep deprivation downregulates dopamine D2R in ventral striatum in the human brain. $J$ Neurosci. 2012;32(19):6711-6717. doi:10.1523/JNEUROSCI.004512.2012

77. Whitney P, Hinson JM, Nusbaum AT. A dynamic attentional control framework for understanding sleep deprivation effects on cognition. Prog Brain Res. 2019;246:111-126. doi:10.1016/bs.pbr.2019.03.015

78. Rausch F, Mier D, Eifler S, et al. Reduced activation in the ventral striatum during probabilistic decision-making in patients in an at-risk mental state. J Psychiatry Neurosci. 2015;40(3):163-173. doi:10. 1503/jpn. 140191

79. Reeve S, Emsley R, Sheaves B, Freeman D. Disrupting sleep: the effects of sleep loss on psychotic experiences tested in an experimental study with mediation analysis. Schizophr Bull. 2018;44 (3):662-671. doi:10.1093/schbul/sbx103

80. Venkatraman V, Chuah YM, Huettel SA, Chee MW. Sleep deprivation elevates expectation of gains and attenuates response to losses following risky decisions. Sleep. 2007;30(5):603-609. doi:10.1093/ sleep/30.5.603

81. Buelow MT. Predicting performance on the Columbia card task: effects of personality characteristics, mood, and executive functions. Assessment. 2015;22(2):178-187. doi:10.1177/1073191114539383

82. Penolazzi B, Gremigni P, Russo PM. Impulsivity and reward sensitivity differentially influence affective and deliberative risky decision making. Pers Individ Dif. 2012;53(5):655-659. doi:10.1016/j.paid.2012.05.018

83. Ferrara M, Bottasso A, Tempesta D, Carrieri M, De Gennaro L, Ponti G. Gender differences in sleep deprivation effects on risk and inequality aversion: evidence from an economic experiment. PLoS One. 2015;10(3):e0120029. doi:10.1371/journal.pone.0120029

84. Greer SM, Goldstein AN, Knutson B, Walker MP. A genetic polymorphism of the human dopamine transporter determines the impact of sleep deprivation on brain responses to rewards and punishments. J Cogn Neurosci. 2016;28(6):803-810. doi:10.1162/jocn_a_00939

85. Smith DV, Hayden BY, Truong TK, Song AW, Platt ML, Huettel SA. Distinct value signals in anterior and posterior ventromedial prefrontal cortex. J Neurosci. 2010;30(7):2490-2495. doi:10.1523/ JNEUROSCI.3319-09.2010

86. Libedinsky C, Smith DV, Teng CS, et al. Sleep deprivation alters valuation signals in the ventromedial prefrontal cortex. Front Behav Neurosci. 2011;5:70. doi:10.3389/fnbeh.2011.00070

87. Menz MM, Buchel C, Peters J. Sleep deprivation is associated with attenuated parametric valuation and control signals in the midbrain during value-based decision making. $J$ Neurosci. 2012;32 (20):6937-6946. doi:10.1523/JNEUROSCI.3553-11.2012
Nature and Science of Sleep

\section{Publish your work in this journal}

Nature and Science of Sleep is an international, peer-reviewed, open access journal covering all aspects of sleep science and sleep medicine, including the neurophysiology and functions of sleep, the genetics of sleep, sleep and society, biological rhythms, dreaming, sleep disorders and therapy, and strategies to optimize healthy sleep.
The manuscript management system is completely online and includes a very quick and fair peer-review system, which is all easy to use. Visit http://www.dovepress.com/testimonials.php to read real quotes from published authors. 